\title{
In reply to 'Cardiotoxicity of the cancer therapeutic agent imatinib mesylate'
}

To the editor:

The recent article by Kerkelä et al. ${ }^{1}$ reported clinical findings of congestive heart failure (CHF) in ten patients, and preclinical studies showing that imatinib-treated mice can develop left ventricular contractile dysfunction and cellular abnormalities suggestive of a toxic myopathy. Many of the patients that developed CHF, however, had pre-existing conditions (as described in their Supplementary Table 1), including hypertension (7 of 10) and diabetes (4 of 10), and three patients had a history of coronary artery disease. Of these three, two required coronary artery bypass grafting and one had a coronary stent in place. Full case reports of these patients were not provided, and so the risks of developing this complication cannot be quantified or placed in the appropriate context on the basis of the findings reported in this article.

We examined the Novartis clinical database of six registration trials, comprising 2,327 patients who received imatinib as monotherapy for one of the following conditions: transformed chronic myelogenous leukemia (CML) or advanced leukemia in blast crisis $(n=553)$, CML in chronic phase (CML-CP) $(n=1442)$, unresectable or metastatic malignant gastrointestinal stromal tumors (GIST) expressing CD117 ( $n=147)$, or a variety of rare malignant diseases $(n=185)$. These trials represent 5,595 years of patient exposure to imatinib (average exposure: 2.4 years). The studies were all conducted in accordance with the Declaration of Helsinki, and the study protocols were reviewed by the Ethics Committees or Institutional Review Boards of all participating centers. All patients gave written informed consent according to institutional regulations. These trials were not specifically designed to capture heart failure events and cardiac ejection fractions were not routinely monitored, but adverse events and serious adverse events were recorded by investigators in all trials. On the basis of the Medical Dictionary for Regulatory Activities (MedDRA), 54 preferred terms were chosen to widely capture $\mathrm{CHF}$ or clinical left ventricular dysfunction events, and the entire clinical database was searched for any adverse events corresponding to these search terms. Full case narratives were developed from this search and reviewed for these events. On the basis of this analysis, 12 cases of CHF $(0.5 \%)$ were considered as incident cases (with no previous history of CHF or left ventricular dysfunction) with a possible or probable relationship to imatinib exposure. If these cases are related to the 5,595 years of imatinib exposure received by patients, then the incidence of $\mathrm{CHF}$ is $0.2 \%$ per year across all trials. In the largest international, randomized phase- 3 study reported to date (refs. 2 and 3), 1,106 patients with newly diagnosed CML-CP were randomized to receive either initial therapy with imatinib or the previous standard treatment of interferon- $\alpha$ plus cytosine arabinoside (IFN + Ara-C). In this study, both treatment options were examined for cardiac safety by Novartis according to an analysis of adverse events as described above. The incident cases of cardiac failure and left ventricular dysfunction, possibly or probably related to exposure to the study medication, was $0.04 \%$ per year ( 1 case in 2,309 years of patient exposure) for patients receiving imatinib versus $0.75 \%$ per year (four cases in 536 years of patient exposure) in patients receiving IFN + Ara-C (data not shown).

Novartis is committed to patient safety and maintains a global safety database that monitors all trials sponsored by Novartis, reports received spontaneously from prescribers and literature reports. These data represent an estimate of more than 200,000 years of patient exposure. Severe congestive heart failure and left ventricular dysfunction, although uncommon,

\section{To the editor:}

Kerkelä et al. ${ }^{1}$ suggest that imatinib treatment could result in cardiotoxicity. Electrocardiography data derived from ten patients, shown in their Figure 1a, demonstrate a decrease in ejection fraction during treatment with imatinib. have occasionally been reported, and cardiac failure is listed on product labeling worldwide. Patients with known cardiac disease or risk factors for cardiac failure should be monitored carefully, and any patient with symptoms consistent with cardiac failure should be evaluated and treated. In an upcoming study, Novartis will evaluate the rate of left ventricular ejection fraction dysfunction, both clinically and subclinically.

To date, imatinib has brought molecularly targeted therapy to patients with lifethreatening diseases, including CML, Philadelphia-positive acute lymphocytic leukemia, and metastatic or inoperable GIST expressing the CD117 antigen. Imatinib has greatly improved the benefit-to-risk ratio compared to previous therapies for these diseases. The observations of Kerkelä et al. must be placed in context, as they are mainly preclinical in focus and the clinical findings have not been fully described.

\section{Alan Hatfield ${ }^{1}$, Samantha Owen ${ }^{2}$ \& Paul Richard Pilot ${ }^{3}$ \\ ${ }^{1}$ Clinical Research, ${ }^{2}$ Global Medical Affairs, and ${ }^{3}$ Integrated Safety, Novartis Pharmaceuticals Corporation, 1 Health Plaza, East Hanover, New Jersey 07936, USA. e-mail: Samantha_jane.owen@novartis.com}

\section{COMPETING INTERESTS STATEMENT}

The authors declare competing financial interests (see the Nature Medicine website for details).

1. Kerkelä, R. et al. Nat. Med. 12, 908-916 (2006).

2. O'Brien, S.G. et al. N. Engl. J. Med. 348, 994-1004 (2003).

3. Druker, B.J. New Engl. J. Med. 355, 2408-2417 (2006)

However, the authors do not provide some needed information: what is the size of the total population from which these ten patients were selected, and did the decrease in ejection fraction happen during treatment with imatinib or after its discontinuation (and, if the latter, how long 